Jamil Dauda Usman*, Mikail Umar Isyaku and Adesoji Adedipe Fasanmade

\title{
Evaluation of heart rate variability, blood pressure and lipid profile alterations from dual transceiver mobile phone radiation exposure
}

https://doi.org/10.1515/jbcpp-2020-0047

Received February 10, 2020; accepted August 2, 2020; published online November 4, 2020

\section{Abstract}

Objectives: Electromagnetic fields have been reported to alter electrical activities in the brain and heart. However, there is paucity of information on the potential functional alterations that magnetic fields from mobile phone could cause to the heart. This study investigated heart rate variability (HRV), blood pressure (BP) and lipid profile in Wistar rats exposed to electromagnetic field radiation from a dual transceiver mobile phone (DTrMP).

Methods: Twenty-one male albino Wistar rats (140-180 g) were randomly assigned to two major groups positioned $5 \mathrm{~m}$ apart as follows: control: no phone $(\mathrm{n}=7)$ and treatment group $(n=14)$ continuously exposed to electromagnetic field from Tecno T312 DTrMP 900/1800 MHz set in silence mode. Experimental treatment consisted in 10 min calls/ day, directed to this device for a period of six weeks. Seven animals from the treatment group were allowed to recover for a period of two weeks after exposure. HRV, systolic, diastolic and mean arterial BP were noninvasively investigated, while serum lipid profile and heart tissue nitric oxide (NO) activities were determined using standard procedures.

Results: There was significant $(\mathrm{p}<0.05)$ increase in systolic, diastolic, mean arterial BP and a decrease in HRV. Serum high density lipoproteins decreased, while total cholesterol, atherogenic indices, and heart NO levels increased significantly in the radiation exposed animals. The alterations observed in exposed animals remained unchanged even after the recovery period.

*Corresponding author: Jamil Dauda Usman, Department of Human Physiology, College of Medicine and Health Sciences, Federal University Dutse, Nigeria; and Department of Physiology, College of Medicine, University of Ibadan, Nigeria, E-mail: jams085@yahoo.com Mikail Umar Isyaku, Department of Human Anatomy, College of Medicine and Health Sciences, Federal University Dutse, Dutse, Nigeria

Adesoji Adedipe Fasanmade, Department of Physiology, College of Medicine,University of Ibadan, Ibadan, Nigeria
Conclusions: These results suggest that exposure to electromagnetic radiation from dual transceiver mobile phones could be a risk factor to increase in blood pressure.

Keywords: blood pressure; heart rate variability; lipid profile; mobile phone electromagnetic radiation.

\section{Introduction}

The use of multiple transceiver mobile phones has become rampant in our society with little or no concern about their potential health risks, especially on the cardiovascular system [1]. Mobile phone subscription rate is about 7.8 billion worldwide as at December 2018 [2]. The increase in subscription rate is probably related to the emergence of dual transceiver mobile phones. Unlike the conventional mobile phones meant to house a single subscriber identification modules (SIM), multiple transceiver phones offer more flexibility to users owing to their increased SIM capacity $[1,3]$. The dual transceivers mobile phones (DTrMP) are known to emit higher electromagnetic energies than their single transceiver prototype [1, 3, 4].

Electromagnetic fields have been reported to alter the electrical activities in the brain [5] and heart [6]. The alterations in the autonomic nervous system are known to induce changes in blood pressure variability [7]. Previous studies have revealed that radiofrequency energies emissions similar to mobile phone radiations can alter biological responses, and references were made to oxidative stress effects $[1,3,8,9]$. Recently, exposure to electromagnetic fields from multiple transceiver mobile phones has been reported to induce anxiolitic behavior, oxidative stress in the serum, brain and heart tissue in rodents $[1,3]$. Furthermore, chronic exposure to this device with at least $10 \mathrm{~min} /$ day exposure rate has also been reported to cause disturbance in sleep cycle. This could lead to alterations in cardiovascular system responses $[1,3,10]$.

High levels of lipid in the serum have been reported to be a very strong risk factor to cardiovascular system dysfunctions. The alterations in some of the lipid profile variable can cause elevation of blood pressure [11, 12]. Nitric oxide (NO) as endothelial tissue relaxing signaling factor 
had also been reported to exert some role in the regulation of lipid metabolism and blood pressure by dilating blood vessels [11-13]. Inhibition of this signaling agent (NO) could lead to accelerated atherosclerosis [11, 14]. Therefore, the current study investigates the effects of exposure to electromagnetic radiation from dual transceiver mobile phones on heart rate variability (HRV), blood pressure (BP) and lipid profile in Wistar rats' model.

\section{Materials and methods}

\section{Materials}

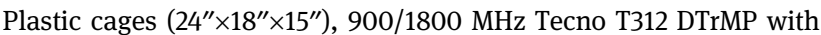
whole body SAR values of $0.851 \mathrm{~W} / \mathrm{kg}$, mobile phone fitted sized wooden box (4.5" $\times 2$ " $\times 0.7$ "), electrocardiography heart rate variability machine by Edan Scientific ${ }^{\circledR}$, China and blood pressure machine by Kent Scientific ${ }^{\circledR}$, USA were used.

\section{Experimental protocol}

Twenty-one (21) 7-8 weeks old male albino Wistar rats (140-180 g) were randomly assigned to two major groups as follows: Control: no phone ( $\mathrm{n}=7$ animals) and treatment group: with electromagnetic field radiation exposure from $\mathrm{DTrMP}(\mathrm{n}=14$ animals). The animals were kept freely at room temperature $\left(25^{\circ} \mathrm{C}\right)$ and humidity of $40 \pm 10 \%$ at $12 \mathrm{~h}$ light/dark cycle condition. They were fed with standard chow and water ad libitum in their respective cages positioned at a distance of $5 \mathrm{~m}$ apart. Animals in the treatment group were continuously subjected to electromagnetic field radiation from a Tecno T312 DTrMP set in silence mode. The phone works on a 900/1800 MHz GSM network with body SAR value of $0.851 \mathrm{~W} / \mathrm{kg}$, and the actual emission during the experiments were not measured. The phone was placed beneath their cages in a fitted sized wooden box [1] as shown in Figure 1.1 below. In order to facilitate comparison with previous studies [1, 3], exposure lasted for 6 weeks with at least 10 min calls/day. After 6 weeks of exposure, seven animals each from the DTrMP electromagnetic field radiation exposed and control groups were assessed for blood pressure and heart rate variability (HRV) as described below. Thereafter, the animals were humanely sacrificed and their tissues were collected for biochemical analyses. The mobile phone was removed from the cage, and the remaining seven animals in the DTrMP electromagnetic field radiation exposed group were kept in the same conditions of initially sacrificed animals for two weeks, after which they were assessed for the experiments listed above. This aspect of the experiment was meant to ascertain whether potential changes due to exposure would be reversed during this period.

Blood pressure (BP) measurement: Blood pressures (BP) of the animals were measured non-invasively using a rat tail cuff connected to volume pressure sensor on a CODA blood pressure machine. Each animal was confined in a cone restrainer, and placed on a

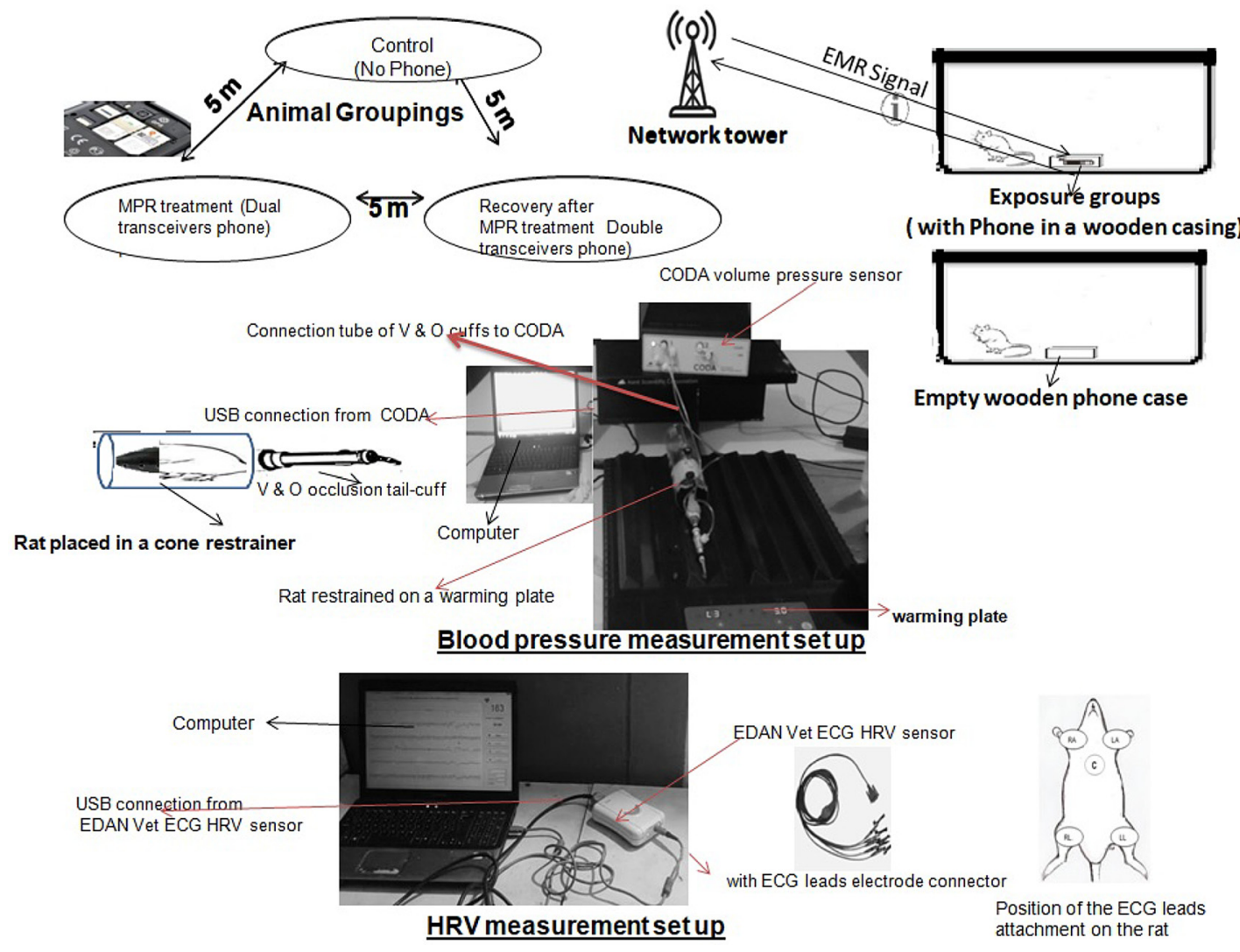

Figure 1.1: Experimental protocol. 


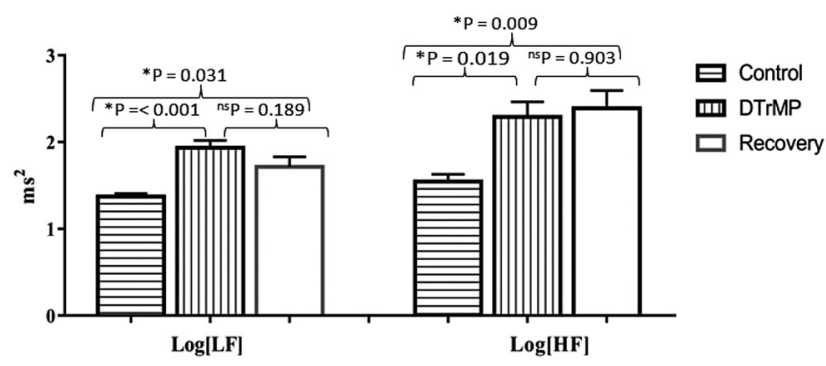

Figure 1: Frequency domain of HRV results of Wistar rats after 6 weeks of exposure to electromagnetic field radiation from a DTrMP. DTrMP: Dual transceiver mobile phone electromagnetic field radiation; ${ }^{*} p<0.05 ;{ }^{n s} p>0.05$.

manually-controlled warming plate with their tail inserted into the $\mathrm{V}$ and $O$ cuff of the machines' transducer. Diastolic BP, systolic BP and mean arterial BP were monitored through a transducer connected to computer via data acquisition system (CODA 2095). Blood pressure variables $(\mathrm{mmHg})$ were recorded trice/session and mean value per session was taken [11].

Electrocardiographic heart rate variability (ECG HRV) studies: Animals from each group were anesthetized with intraperitoneal injection of $0.1 \mathrm{ml} / 100 \mathrm{~g} 40 \mathrm{mg} / \mathrm{ml}$ xylazine and $25 \mathrm{mg} / \mathrm{ml}$ of ketamine hydrochloride (1:1). ECG HRV lead electrode of the ECG HRV machine were placed in their respective position on the chest, right arms, right legs, left arms and left legs of the animals. The HRV data were monitored electrocardiographically for $5 \mathrm{~min}$ on a computer connected to the ECG HRV transducer as described in Figure 1.1 below. The variable measured in the frequency domain of the HRV were low frequency variation of $R R$ intervals (LF) and high frequency variation of $R R$ intervals (HF), while average time between consecutive $\mathrm{R}$ waves (RR interval), ratio of maximum to minimum values of RR interval (Max/ Min ratio) and root mean square of successive difference between adjacent RR intervals (RMSSD) were recorded on the time domain [15].

Serum lipid profile studies: Blood samples were collected through intracardiac puncture into plain sample bottles and centrifuged for $10 \mathrm{~min}$ at $5000 \mathrm{rpm}$. The serum was decanted for high density lipoprotein (HDL), triacylglycerol (TG) and total cholesterol (TC) biochemical assay using the procedure highlighted in the commercially available kits by Fortress diagnostics, Antrim, UK. Low-density lipoprotein cholesterol (LDL) concentration was estimated using
$\mathrm{LDL}=\mathrm{TC}-(\mathrm{HDL})-(\mathrm{TG} / 5)$, while atherogenic indices was calculated using $(\mathrm{AI})=[\mathrm{TC}-\mathrm{HDL}] /[\mathrm{HDL}][16]$.

Heart tissue nitric oxide (NO) assay: The heart of each animal was dissected and homogenized ( $1 \mathrm{~g}$ of tissue per $4 \mathrm{ml}$ ) in $0.1 \mathrm{M}$ phosphate buffer of $\mathrm{pH} 7.4$ at $4^{\circ} \mathrm{C}$. The homogenized tissue was centrifuged using a cold centrifuge machine at $10000 \mathrm{rpm}$ to get a clear tissue fluid for the NO biochemical assay. Griess reagent method was used to quantify nitrites/nitrates activities in the homogenate $[1,17]$.

\section{Statistical analysis}

Data were subjected to analysis of variance (ANOVA) using GraphPad Prism 7. Mean comparison was done using Tukey HSD test $(\mathrm{p}<0.05)$. Results was expressed as mean \pm SEM.

\section{Results}

\section{Blood pressure results}

Systolic BP, diastolic and mean arterial BP increased significantly in animal exposed to DTrMP electromagnetic field radiation and recovery group compared to their control $(\mathrm{p}<0.05)$. Heart rate values were not significantly different when animals of the DTrMP electromagnetic field radiation exposed and recovery groups were compared to control. Systolic BP, diastolic BP, mean arterial BP and heart rate values of animals in the recovery group were not significantly different from the values of the DTrMP electromagnetic field radiation exposed group (Table 1).

\section{Heart rate variability results}

\section{Time domain of HRV}

RR interval and RMSSD values of animal in both the DTrMP electromagnetic field radiation exposed and recovery groups were not significantly different from their control

Table 1: Systolic, diastolic, mean arterial BP $(\mathrm{mmHg})$ and heart rate (beats/min) results of Wistar rats after 6 weeks of exposure to electromagnetic field radiation from a DTrMP.

\begin{tabular}{lrrrrrr}
\hline & Control & DTrMP & Recovery & $\begin{array}{r}\text { p-Value control } \\
\text { vs DTrMP }\end{array}$ & $\begin{array}{r}\text { p-Value control } \\
\text { vs recovery }\end{array}$ & $\begin{array}{r}\text { p-Value DTrMP } \\
\text { vs recovery }\end{array}$ \\
\hline Systolic BP & $128.70 \pm 2.80$ & $186.30 \pm 8.41$ & $201.00 \pm 12.52$ & $0.002^{\star}$ & $<0.001^{\star}$ & $0.488^{\text {ns }}$ \\
Diastolic BP & $91.38 \pm 3.64$ & $136.30 \pm 6.42$ & $122.5 \pm 9.62$ & $0.002^{\star}$ & $0.021^{\star}$ & $0.374^{\text {ns }}$ \\
MABP & $103.50 \pm 2.82$ & $152.60 \pm 6.06$ & $148.00 \pm 2.24$ & $<0.001^{\star}$ & $<0.001^{\star}$ & $0.712^{\text {ns }}$ \\
Heart rate & $396.10 \pm 11.73$ & $421.70 \pm 24.14$ & $328.00 \pm 38.80$ & $0.794^{\text {ns }}$ & $0.232^{\text {ns }}$ & $0.081^{\text {ns }}$ \\
\hline
\end{tabular}

DTrMP, Dual transceiver mobile phone electromagnetic field radiation; BP, Blood pressure; MABP, Mean arterial blood pressure; ${ }^{*} p<0.05 ;{ }^{\text {ns }} p>0.05$ 
values. A significant decrease was observed in the max/ min values of RR interval ratio (RR Max:Min) of animals exposed to DTrMP electromagnetic field radiation compared to control $(\mathrm{p}=<0.001)$, while no significant differences were observed in the values of RR max:min of the recovery group compared to control. RR max:min values significantly increase $(\mathrm{p}=<0.001)$, while no significant changes was observed in the RR interval and RMSSD when their values in animals from the recovery group were compared to the DTrMP electromagnetic field radiation exposed group (Figure 2).

\section{Frequency domain of HRV}

Values of LF and HF increased significantly in animals exposed to DTrMP electromagnetic field radiation and recovery groups when compared to their controls. LF and $\mathrm{HF}$ values of animals in the recovery group were not significantly different from the values of the DTrMP electromagnetic field radiation-exposed group (Figure 1).

\section{Lipid profile}

Serum high density lipoprotein (HDL) and total cholesterol (TC) levels decreased significantly, while atherogenic indices (AI) increased significantly when their values in animals exposed to DTrMP electromagnetic field radiation were compared to the control. TG and LDL levels were not significantly different in the DTrMP electromagnetic field radiation exposed animals and the recovery group compared to control. TC and HDL levels were not significant, while AI increased significantly when their values in animals of the recovery group were compared to control. HDL, LDL, TC, TG and AI levels in animals of the recovery group were not significantly different from the values of the DTrMP electromagnetic field radiation exposed group (Figures 3, 4, and 5).

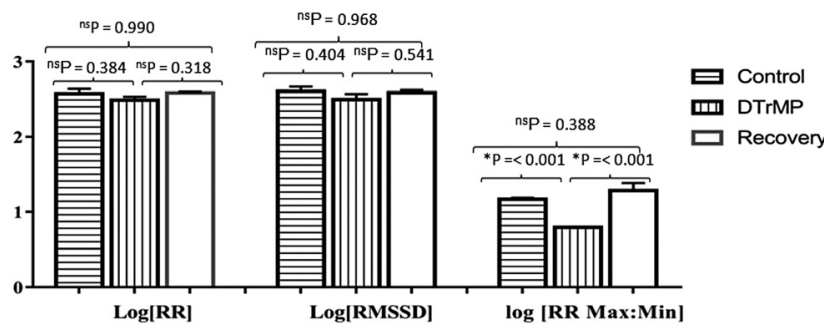

Figure 2: Time domain of HRV results of Wistar rats after 6 weeks of exposure to electromagnetic field radiation from a DTrMP. DTrMP: Dual transceiver mobile phone electromagnetic field radiation; ${ }^{\star} p<0.05 ;{ }^{n s} p>0.05$.

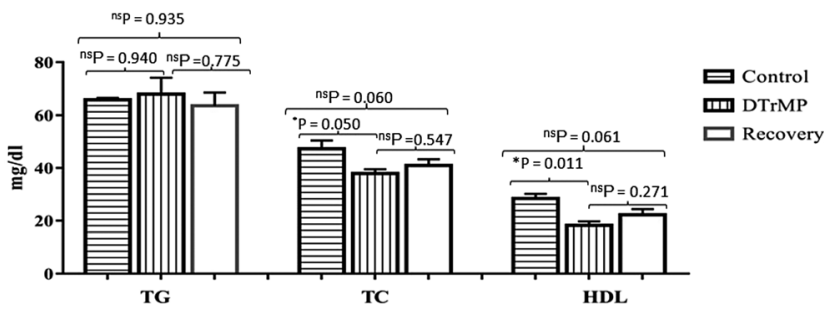

Figure 3: Serum high density lipoprotein (HDL), triglycerides (TG) and total cholesterol (TC) results of Wistar rats after 6 weeks of exposure to electromagnetic field radiation from a DTrMP. DTrMP: Dual transceiver mobile phone electromagnetic field radiation; ${ }^{*} p<0.05 ;{ }^{n s} p>0.05$.

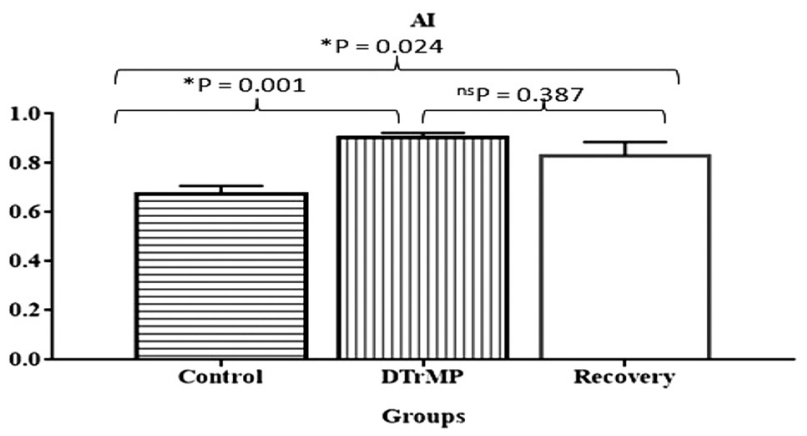

Figure 4: Serum atherogenic indices (Al) results of Wistar rats after 6 weeks of exposure to electromagnetic field radiation from a DTrMP. DTrMP: Dual transceiver mobile phone electromagnetic field radiation; ${ }^{*} p<0.05 ;{ }^{n s} p>0.05$.

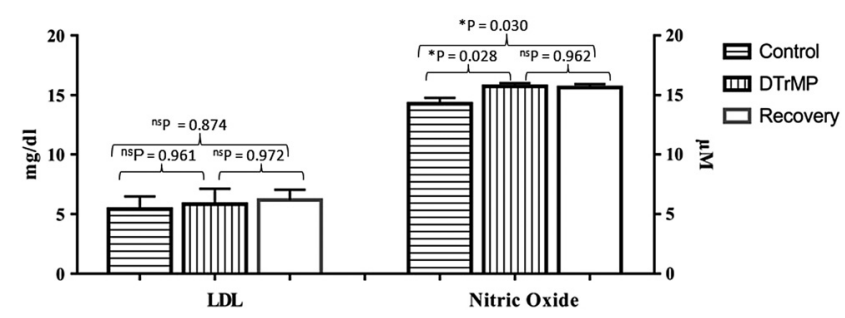

Figure 5: Serum low density lipoprotein (LDL) and nitric oxide activities results of Wistar rats after 6 weeks of exposure to electromagnetic field radiation from a DTrMP. DTrMP: Dual transceiver mobile phone electromagnetic field radiation; ${ }^{*} p<0.05$; ${ }^{\text {ns }} \mathrm{p}>0.05$.

\section{Nitric oxide (NO) activity}

Heart tissue nitric oxide from animals in both the DTrMP electromagnetic field radiation-exposed and recovery groups significantly increased compared to control $(p<0.05)$, while it values in animals of the recovery group were not significantly different from that of DTrMP electromagnetic field radiation exposed group (Figure 5). 


\section{Discussions}

Findings from this study present that exposure to electromagnetic field radiation from dual transceiver mobile phone significantly increased the level of systolic, diastolic and mean arterial blood pressure. This could be as result of increase in serum lipid profile that resulted into increased level of atherogenic indices which can potentially increase peripheral resistance, or increase in the sympathetic drive to the heart musculature that could possibly cause an increase in the stroke volume. Variations in the systolic, diastolic and mean arterial blood pressures had been reported in previous studies to be directly proportional to variation in the stroke volume and tissue peripheral resistance $[18,19]$. This report on blood pressure is also consistent with the findings of Saili et al. [20] who reported an increase in the arterial blood pressure in rabbits exposed to $2.45 \mathrm{GHz}$ of wireless fidelity (WIFI) radiations. However, they associated the observed increase in blood pressure to the direct action of radiofrequency on $\mathrm{Ca}^{2+}$ and $\mathrm{Zn}^{2+}$ homeostasis alterations, rather than the increase in lipid profile as observed in the current study. Braune et al. [21] have also observed that $35 \mathrm{~min}$ exposure to Global System for Mobile communication simulated signal increased blood pressure by $10 \%$ in volunteer individuals, while Thomas and Tenforde [22] illustrated a rise in the blood pressure of individuals working under magnetic field environment.

The present study also showed that electromagnetic field exposure from dual transceiver mobile can alter some variables in heart rate variability (HRV) via changes in the electrical activities of the heart. In the time domain of the $\mathrm{HRV}$, the average time between consecutive $\mathrm{R}$ waves (RR interval), ratio of maximum to minimum values of $R R$ interval and RMSSD in heart electrocardiogram decreased in the radiation-exposed group. These observations were similar to the report of Bortkiewicz et al. [23] investigating radio and TV transmission workers. Increased LF and HF levels obtained in our study agree with the findings of Andrzejak et al. [24] and Havas et al. [25] on the effects of radiofrequency electromagnetic field exposure in humans.

High levels of serum HDL and atherogenic indices in the present study suggest dyslipidemia [29]. These observations are similar to the reports of Luo et al. in their study of electromagnetic fields exposure to rats [27]. The nonsignificant increase in serum LDL observed is concordant with the study of Güler et al. [28] where they exposed guinea pigs to different intensities and duration of electromagnetic fields.

The variation of serum lipids observed in the present study can be as a result of electromagnetic field ability to penetrate and act on cells by changing their membrane potential, which leads to alterations in the serum biochemical processes [29,30], or alterations through oxidative stress mechanism and inflammatory cells responses [1]. High $(35 \mathrm{GHz})$ or low $(50 \mathrm{~Hz})$ frequency electromagnetic field radiations have been reported to also trigger inflammatory response of macrophages from the bone marrow of rats [31,32]. The responding inflammatory cells can enter the blood vessel and take up cholesterol with other fatty substances to build up plaques forming arteriosclerosis [33], thereby causing an increase in the blood pressure observed. The increased level of NO activities in the heart can be due to compensatory response to increase in blood pressure [11] or inflammatory response in the cardiomyoctes [31].

Inability of the blood pressure to recover despite the decrease in heart rate and increased levels of $\mathrm{NO}$ activity in the heart could be as a result of increased peripheral resistant resulting from increase in lipogenesis and atherogenic indices, or due to increase level of stroke volume resulting from increase in the force of myocadial contraction which are factors known to cause increase in the arterial blood pressure [18] observed in the present studies. More so, it could also result from loss in the sensitive complex neurocardiac control [34] resulting from the increased level of LF, HF and decreased RMSSD values that leads to decrease in the heart rate variability reported in the present work. Thus, this enhanced sympathetic drive caused by direct interaction of electromagnetic fields radiated from mobile phone with the electrical activities of the heart $[1,35]$. The enhanced sympathetic activities could also be as a result of increased oxidative stress and anxiety as previously reported $[1,3,36]$.

The limitation to present study was the inability to measure energy density inside the cages where the animals were placed with the mobile phone. However, all experiments were performed in a quite laboratory with zero interference to charges from other electric appliances. Furthermore, energy density in a space, room or the laboratory environment, where the experiment were conducted in reality would be too difficult to monitor. This situation emanates from fluctuations in the strengths of signal transmissions all the time of the day from communication towers of the network provider to transceivers on the mobile phone.

\section{Conclusions}

This study suggests that exposure to radiation from dual transceiver mobile phones electromagnetic fields could be 
a risk factor to rise in blood pressure in cardiovascular system dysfunction of unknown pathology.

Acknowledgments: The authors' acknowledgment goes to members of cardio-respiratory Unit of physiology department, Small Animal Clinic of Veterinary Medicine Department, University of Ibadan for their technical support.

Research funding: None declared.

Author statements: All authors have accepted responsibility for the entire content of this manuscript and approved its submission.

Competing interests: Authors declare no conflict of interests.

Ethical approval: All procedures involved in the animal handling and protocol of the experiments, were strictly in accordance to the ethics and guidelines of animal care and use for research (UI - ACUREC, 2018), University of Ibadan and regulations governing care and use of experimental animals (NIH Pub. No. 85-23 revised 1999).

\section{References}

1. Usman JD, Isyaku UM, Magaji RA, Fasanmade AA. Assessment of electromagnetic fields, vibration and sound exposure effects from multiple transceiver mobile phones on oxidative stress levels in serum, brain and heart tissue. Sci Afr 2020. https://doi. org/10.1016/j.sciaf.2020.e00271 [Epub ahead of print].

2. ITU https://www.itu.int/en/ITU-D/Statistics/Documents/ statistics/2018/Fixed_tel_2000-2017_Dec2018.xls.

3. Shehu A, Mohammed A, Magaji RA, Muhammad MS. Exposure to mobile phone electromagnetic field radiation, ringtone and vibration affects anxiety-like behaviour and oxidative stress biomarkers in albino wistar rats. Metab Brain Dis 2016;31:355-62.

4. Hyland GJ. Physics and biology of mobile telephony. Lancet 2000; 356:1833-6.

5. Consales C, Merla C, Marino C, Benassi B. Electromagnetic fields, oxidative stress, and neurodegeneration. Int I Cell Biol 2012; 2012. https://doi.org/10.1155/2012/683897.

6. Barutcu I, Esen AM, Kaya D, Turkmen M, Karakaya O, Saglam M, et al. Do mobile phones pose a potential risk to autonomic modulation of the heart? Pac Clin Electrophysiol 2011;34:1511-4.

7. Grippo AJ. Mechanisms underlying altered mood and cardiovascular dysfunction: the value of neurobiological and behavioral research with animal models. Neurosci Biobehav Rev 2009;33:171-80.

8. Friedman J, Kraus S, Hauptman Y, Schiff Y, Seger R. Mechanism of short-term ERK activation by electromagnetic fields at mobile phone frequencies. Biochem J 2007;405:559-68.

9. Sirav B, Seyhan N. Effects of GSM modulated radio-frequency electromagnetic radiation on permeability of blood-brain barrier in male \& female rats. J Chem Neuroanat 2016;75:123-7.

10. Cabré-Riera A, Torrent M, Donaire-Gonzalez D, Vrijheid M, Cardis E, Guxens M. Telecommunication devices use, screen time and sleep in adolescents. Environ Res 2019;171:341-7.
11. Aluko EO, Omobowale TO, Oyagbemi AA, Adejumobi OA, Ajibade TO, Fasanmade AA. Reduction in nitric oxide bioavailability shifts serum lipid content towards atherogenic lipoprotein in rats. Biomed Pharmacother 2018;101:792-7.

12. Dharmashankar K, Widlansky ME. Vascular endothelial function and hypertension: insights and directions. Curr Hypertens Rep 2010;12:448-55.

13. Ribiere C, Jaubert AM, Gaudiot N, Sabourault D, Marcus ML, Boucher JL. White adipose tissue nitric oxide synthase: a potential source for NO production. Biochem Biophys Res Commun 1996;222:706-12.

14. Cayatte AJ, Palacino JJ, Horten K, Cohen RA. Chronic inhibition of nitric oxide production accelerates neointima formation and impairs endothelial function in hypercholesterolemic rabbits. Arterioscler Thromb 1994;14:753-9.

15. Stoyell-Conti FF, Santos F, Machi JF, Hernandez DR, Barboza CA, Irigoyen MC, et al. Measurement of mouse heart rate variability using echocardiographic system. J Cardiovascul echograp 2018; 28:90.

16. Suanarunsawat T, Watcharaporn D, Na A, Thanapat S, Suwan T, Somlak P. Lipid-lowering and antioxid.comative activities of aqueous extracts of Ocimum sanctum L. leaves in rats fed with a high-cholesterol diet. Oxi Med Cel Long 2011;2011.

17. Grandati M, Verrecchia C, Revaud ML, Allix M, Boulu RG, Plotkine M. Calcium-independent NO-synthase activity and nitrites/ nitrates production in transient focal cerebral ischaemia in mice. Br J Pharmacol 1997;122:625-30.

18. Jamil M, Alun H. Cardiac and vascular pathophysiology in hypertension. Heart 2003;89:1104-9.

19. Nisha C, Jennifer A R. Sympathetic neural mechanism in human cardiovascular health and diseases. Mayo Clin Proc 2009;84:822-30.

20. Saili L, Hanini A, Smirani C, Azzouz I, Azzouz A, Sakly M, et al. Effects of acute exposure to WIFI signals $(2.45 \mathrm{GHz})$ on heart variability and blood pressure in Albinos rabbit. Environ Toxicol Pharmacol 2015;40:600-5.

21. Braune $S$, Wrocklage $C$. Resting blood pressure increase during exposure to a radio-frequency electromagnetic field. Lancet 1998;351:1857-8.

22. Tenforde TS. Magnetically induced electric fields and currents in the circulatory system. Prog Biophys Mol Biol 2005;87: 279-88.

23. Bortkiewicz A, Gadzicka E, Szymczak W, Zmyślony M. Heart rate variability (HRV) analysis in radio and TV broadcasting stations workers. Int J Occup Med Environ Health 2012;25: 446-55.

24. Andrzejak R, Poreba R, Poreba M, Derkacz A, Skalik R, Gac P, et al. The influence of the call with a mobile phone on heart rate variability parameters in healthy volunteers. Ind Health 2008;46: 409-17.

25. Havas M, Jeffrey M, Bernard P, Elizabeth K, Rees CR, Lisa T. Provocation study using heart rate variability shows microwave radiation from $2.4 \mathrm{GHz}$ cordless phone affects autonomic nervous system. Eur J Oncol Lib 2010;5:273-300.

26. Nelson RH. Hyperlipidemia as a risk factor for cardiovascular disease. Prim Care Clin Off Pract 2013;40:195-211.

27. Luo E, Shen G, Xie K, Wu X, Xu Q, Lu L, et al. Alimentary hyperlipemia of rabbits is affected by exposure to low-intensity pulsed magnetic fields. Bioelectromagnetics: J Bioelectromagnetics Soc 2007;28:608-14. 
28. Güler G, Türközer Z, Seyhan N. Electric field effects on Guinea pig serum: the role of free radicals. Electromagn Biol Med 2007;26: 207-23.

29. Coskun O, Comlekci S. Effect of ELF electric field on some on biochemistry characters in the rat serum. Toxicol Ind Health 2011; 27:329-33.

30. Wang Z, Wang L, Zheng S, Ding Z, Liu H, Jin W, et al. Effects of electromagnetic fields on serum lipids in workers of a power plant. Environ Sci Pollut Res 2016;23:2495-504.

31. Kim SJ, Jang YW, Hyung KE, Lee DK, Hyun KH, Jeong SH, et al. Extremely low-frequency electromagnetic field exposure enhances inflammatory response and inhibits effect of antioxidant in RAW 264.7 cells. Bioelectromagnetics 2017;38: 374-85.

32. Vlasova II, Mikhalchik EV, Gusev AA, Balabushevich NG, Gusev $\mathrm{SA}$, Kazarinov KD. Extremely high-frequency electromagnetic radiation enhances neutrophil response to particulate agonists. Bioelectromagnetics 2018;39:144-55.

33. McAlpine CS, Kiss MG, Rattik S, He S, Vassalli A, Valet C, et al. Sleep modulates haematopoiesis and protects against atherosclerosis. Nature 2019;566:383-7.

34. Carney RM, Freedland KE. Depression and heart rate variability in patients with coronary heart disease. Cleve Clin J Med 2009;76:S13.

35. Johansson 0 . Disturbance of the immune system by electromagnetic fields-a potentially underlying cause for cellular damage and tissue repair reduction which could

lead to disease and impairment. Pathophysiology 2009;16:157-77.

36. Martínez-Sámano J, Flores-Poblano A, Verdugo-Díaz L, JuárezOropeza MA, Torres-Durán PV. Extremely low frequency electromagnetic field exposure and restraint stress induce changes on the brain lipid profile of Wistar rats. BMC Neurol 2018;19:31. 